\title{
Sensibilidad al gluten: presentación de tres casos
}

\author{
Gluten sensitivity: presentation of three cases
}

\author{
Dra. Lorena Menéndez ${ }^{a}$, Dra. Luciana Guzmán ${ }^{a}$ Dr. Eduardo Cueto Rúa y Dr. Ricardo Ben ${ }^{a}$
}

\begin{abstract}
RESUMEN
En los últimos años, ha cobrado mayor interés la existencia de un cuadro clínico muy similar al de la enfermedad celíaca, que no se ajusta a los cánones tradicionales de diagnóstico. Se trata de pacientes con una alta sospecha diagnóstica de enfermedad celíaca, que presentan serología y biopsia de intestino delgado normal. La literatura relata, desde la década del 80, la existencia de un síndrome que relaciona el gluten de la dieta con un efecto tóxico generador de síntomas gastrointestinales en presencia de una mucosa normal. A esta entidad se la denominó síndrome de Cooper-Cook. En los últimos años, ha habido numerosas publicaciones que hacen referencia a esta entidad, pero ahora bajo la denominación de sensibilidad al gluten. En el siguiente artículo, se presentan tres casos clínicos que hacen referencia a esta enfermedad.

Palabras clave: sensibilidad al gluten, enfermedad celíaca, alergia al trigo.
\end{abstract}

\section{ABSTRACT}

In the last few years, the existence of a clinical profile similar to celiac disease has become important; this disease does not adapt to the traditional diagnosis canons. It is related to a number of patients who are diagnosed as having the celiac disease but present normal serology and small bowel's biopsy. Since the 80 's, medical literature reports the existence of a syndrome that connects gluten diet with a toxic effect that produces gastrointestinal symptoms even though the mucosa remains normal. This disease is called the Cooper-Cook syndrome. Over the last few years, there have been lots of publications about this disease under the name "gluten sensitivity". In the following article, three clinical cases that refer to this condition are presented.

Key words: gluten sensitivity, celiac disease, wheat allergy.
\end{abstract}

http:/ /dx.doi.org/10.5546/aap.2015.e83

\section{INTRODUCCIÓN}

En la actualidad, se supone que el gluten condiciona múltiples patologías. La más estudiada y mejor conocida es la enfermedad celíaca (EC), que afecta al 1\% de la población, $\mathrm{y}$, con menor frecuencia, la alergia alimentaria al trigo, del 0,4 al 1\%. ${ }^{1}$ Sin embargo, la situación

a. Servicio de Gastroenterología,

Hospital Sor María Ludovica de La Plata.

\section{Correspondencia:}

Dra. Lorena Menéndez: lorena.menendez@hotmail.com

Conflicto de intereses: ninguno que declarar.

Recibido: 11-03-2014

Aceptado: 23-10-2014 patológica causada por la ingesta de gluten más frecuente (se estima que llega a afectar al 6\% de la población) recibe el nombre de sensibilidad al gluten no celíaca. La literatura relata, desde la década del $80,{ }^{2}$ la existencia de un síndrome que relaciona el gluten de la dieta con un efecto tóxico generador de síntomas gastrointestinales en presencia de una mucosa intestinal normal. A esta entidad nuestro grupo la denominó síndrome de Cooper-Cook ${ }^{3}$ para precisar aquellos casos en que las personas mejoraban con una dieta libre de gluten ( $\sin$ TACC: $\sin$ trigo, avena, cebada y centeno). En los últimos años, se han multiplicado las publicaciones en las que se hace referencia a esta entidad, la cual se ha nombrado como sensibilidad al gluten (SG).

En el presente artículo, describimos tres pacientes que se presentaron con esta enfermedad.

\section{PRESENTACIÓN CLÍNICA}

Presentamos tres pacientes de sexo femenino cuyas edades eran de 18 meses, 3 años y 6 años y 8 meses. Los síntomas por los que consultaron fueron distensión abdominal, diarrea crónica, desnutrición y signos carenciales (características en piel y mucosas que evidencian la falta de absorción de micronutrientes, tales como piel seca, caída de cabello, pelo ralo, uñas quebradizas, lengua depapilada, queilitis angular). Por presentar fuerte sospecha de EC, se completó el puntaje diagnóstico de CELIARED (Red Provincial de Celiaquía; véase Figura 1). ${ }^{4}$ Los síntomas de cada uno de los pacientes se resumen en la Tabla 1.

La serología para EC e IgE específica para trigo en todos los casos fue negativa. La biopsia intestinal fue normal con ausencia de atrofia vellositaria. En dos de los pacientes, se realizó el estudio genético (HLA DQ2-DQ8), con resultados negativos.

En todos los casos, se realiza tratamiento antiparasitario.

Por persistencia de los síntomas clínicos, se realizó una prueba de exclusión al gluten durante cuatro semanas. Todos los pacientes respondieron favorablemente con normalización de las deposiciones y aumento de peso. Para 


\section{Malseriode 1 Buenos Aires}

\section{Celiared - Red Provincial de la Celiaquía}

CRITERIOS CLINICOS Y SEROLOGICOS PARA SU SOSPECHA Y REGISTRO

Dirección Provincial de Medicina Preventiva - Dirección de Patologías Prevalentes

\begin{tabular}{|c|c|c|c|c|c|c|c|c|c|c|}
\hline \multicolumn{4}{|c|}{ Apellido y nombre } & \multicolumn{3}{|l|}{ Documento } & \multirow{2}{*}{\multicolumn{4}{|c|}{$\begin{array}{l}\text { Sexo: } F \square \quad M \square \\
\text { Código Postal }\end{array}$}} \\
\hline \multirow{2}{*}{\multicolumn{4}{|c|}{$\begin{array}{l}\text { Fecha de Nacimiento } \\
\text { Hospital de asistencia }\end{array}$}} & Localidad/par & rtido & & & & & \\
\hline & & & & Auto Anticuerpos: & Positivo $\square$ & Nega & itivo $\square$ & No & se hizo & \\
\hline Endoscopia: & & Biop & Clas & de Drut. & Grado & $0 \square$ & $1 \square$ & $2 \square$ & $3 \square$ & $4 \square$ \\
\hline Endustopia. & $\begin{array}{l}\square \text { 1-Nodular } \\
\square \text { 2-Peine }\end{array}$ & & ó Clasif & de Marsh & Grado & $1 \square$ & $2 \square$ & $3 A \square$ & $3 \mathrm{~B} \square$ & $3 c$ \\
\hline & $\begin{array}{l}\text { 3-Scalloping } \\
\text { 4-Perdida pliegues }\end{array}$ & & Año de di & nóstico & Mes de diag & |nóstico & & & & \\
\hline
\end{tabular}

\begin{tabular}{llll}
\hline Diagnóstico Previo de Celiaquía: & SI $\square \quad$ NO $\square \quad$ Celiaco: SI $\square \quad$ NO $\square$ \\
\hline
\end{tabular}

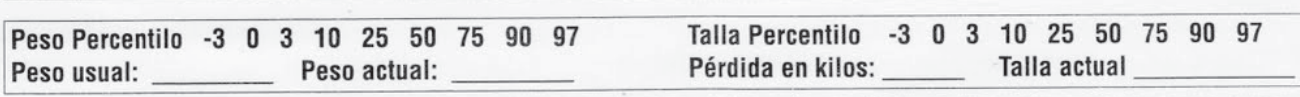

\begin{tabular}{|c|c|c|c|c|c|}
\hline $\begin{array}{c}\text { MAYORES } \\
\text { Consignar solo los positivos }\end{array}$ & $\begin{array}{c}4 \text { ptos } \\
\text { c/u }\end{array}$ & $\begin{array}{l}\text { INCLUYENTES } \\
\text { Consignar solo los positivos }\end{array}$ & $\begin{array}{c}4 \mathrm{ptos} \\
\mathrm{C} / \mathrm{u}\end{array}$ & $\begin{array}{c}\text { MENORES } \\
\text { Consignar solo los positivos }\end{array}$ & $\begin{array}{c}3 \text { ptos } \\
\mathrm{c} / \mathrm{u}\end{array}$ \\
\hline 1 Diarrea Crónica & & 1 Deficit Inmune. & & 1 Flatos fétidos & \\
\hline 2 Desnutrición & & ¿Cuál? & & 2 Nauseas & \\
\hline 3 Distensión Abdominal & & 2 Diabetes Tipo & & 3 Dolor Abdominal recurrente & \\
\hline 4 Signos Carenciales & & 3 Sindrome De Down & & 4 Astenia & \\
\hline 5 Baja Talla Comparativa & & 4 Colagenopatías & & 5 Irritabilidad & \\
\hline 6 Abdomen Inferior Mate & & 5 Hepatitis Autoinmune & & 6 Trast. de conducta & \\
\hline 7 Pruebas Lab. Alteradas & & 6 Hipotiroidismos & & 7 Pariente Celíaco en $2^{\circ}$ grado & \\
\hline 8 IgG AGA, (antigliadina) & & 7 Hipertiroidismo & & 8 Artromialgias & \\
\hline $9 \lg A$ AGA & & 8 Nefropatía dep. $\lg A$ & & 9 Retraso puberal & \\
\hline 10 Prolapso & & 9 TGO y/o TGP (no virales) & & 10 Vómitos & \\
\hline 11 Alteración del esmalte & & 10 Calcific. Cerebrales & & 11 Cefaleas & \\
\hline 12 Edad Osea < de 2 años & & 11 Enf. De Duhring & & 12 Plenitud & \\
\hline 13 Edemas & & 12 Trast. Neurológicos & & & \\
\hline 14 Anemia crónica & & 13 Depresión & & & \\
\hline 15 Anemia $x$ déficit de hierro & & 14 Autismo & & EXCLUSIVOS & 35 ptos \\
\hline 16 Anemia $x$ déficit de folatos & & 15 Hiperactividad & & Consignar solo los positivos & $\mathrm{c} / \mathrm{u}$ \\
\hline 17 Anemia $\because$ déficit de Vit. grupo B & & 16 Déficit atención & & 1 IgA Ema (antiendomisio) & \\
\hline 18 Osteoporosis & & 17 Ataxia & & 2 IgA tTG (transglutaminasa) valor: & \\
\hline 190 sl zopenia & & 18 Psoriasis & & 3 IgA AGA 2 valor: & \\
\hline 20 Aburtos & & 19 Vitiligo & & 4 Test rápido & \\
\hline 21 Inipotencia & & 20 Púrpura trombocitopenica & & 5 IgG Ema (antiendomisio) & \\
\hline 22 Pariente Celíaco en $1^{\circ}$ grado & & 21 Alopecías & & 6 IgG tTG (transglutaminasa) valor: & \\
\hline 23 Hermanos Eutróficos & & 22 Fliar c/enf. Autoinmune & & 7 IgG AGA (antigliadina) 2 valor: & \\
\hline $24 \mathrm{Dq} 2$ & & 23 Síndrome de Sjögren & & & \\
\hline $25 \mathrm{Dq} 8$ & & 24 Síndrome de Turner & & & \\
\hline SUMA MAYORES & & SUMA INCLUYENTES & & SUMA MENORES & \\
\hline SUMA CLINICA & & SUMA EXCLUSIVOS & & SUMA TOTAL & \\
\hline
\end{tabular}

\begin{tabular}{|l|c|c|c|c|c|c|}
\hline SUMATORIA DE PUNTAJE CLINICO & 8 puntos & $12 p$ & $20 p$ & $24 p$ & $32 p$ & $50 p 0$ mas \\
\hline PROBABILIDAD DE CELIAQUIA & $10 \%$ & $20 \%$ & $40 \%$ & $50 \%$ & $80 \%$ & $100 \%$ \\
\hline
\end{tabular}

Comentario Final:

Consultas adultos Dres. J C. Gomez, A Crivelli, Lic. Nutr. A. Baistrocchi: unidad@soportenutricional.com.ar Consultas pediátricas Dres E A. Cueto Rua, L. Guzman, G. Nanfito: hmlgastro@intramed.net.ar Consultas Programa Celiaquía: celiared@ms.gba.gov.ar 
confirmar el diagnóstico, se realizó una prueba de provocación, en la que se evidenció la reaparición de la sintomatología (Tabla 1).

\section{DISCUSIÓN}

El gluten es la principal proteína estructural compleja del trigo. Presenta equivalentes tóxicos en otros cereales, como la cebada y el centeno, que incluyen las gliadinas y gluteninas. Posiblemente, la introducción de granos que contenían gluten hace más de 10000 años con el advenimiento de la agricultura representó un desafío evolutivo que creó las condiciones necesarias para el desarrollo de las enfermedades relacionadas con la exposición al gluten, dentro de ellas la enfermedad celíaca, la alergia al trigo y la sensibilidad al gluten.
Definimos la sensibilidad al gluten en aquellos casos de reacción al gluten en los que ambos mecanismos (alérgico y autoinmune) pueden ser descartados. ${ }^{5}$

Más específicamente, son aquellos casos en que ocurre lo siguiente:

- Los tests inmunoalérgicos al trigo o la serología son negativos para EC;

- Se descarta el déficit de IgA;

- Histopatología intestinal normal;

- Posible presencia de biomarcadores de reacciones nativas al gluten (antigliadina de primera generación -AGA- +);

- Síntomas clínicos solapados con los síntomas de enfermedad celíaca y alergia al trigo;

- Pacientes que muestran resolución de sus síntomas cuando comienzan una dieta sin TACC.

TABLA 1.

\begin{tabular}{|c|c|c|c|}
\hline & Paciente 1 & Paciente 2 & Paciente 3 \\
\hline Edad & 3 años & 6 años y 8 meses & 1 año y 6 meses \\
\hline Sexo & Femenino & Femenino & Femenino \\
\hline $\begin{array}{l}\text { Criterios mayores } \\
\text { ("signos") }\end{array}$ & $\begin{array}{c}\text { Signos carenciales } \\
\text { Distensión abdominal } \\
\text { Diarrea crónica }\end{array}$ & $\begin{array}{c}\text { Diarrea crónica } \\
\text { Distensión abdominal } \\
\text { Desnutrición. Signos carenciales } \\
\text { Alteración del esmalte dentario }\end{array}$ & $\begin{array}{c}\text { Diarrea crónica } \\
\text { Distensión abdominal } \\
\text { Signos carenciales } \\
\text { Desnutrición }\end{array}$ \\
\hline $\begin{array}{l}\text { Criterios menores } \\
\text { ("síntomas") }\end{array}$ & & Flatos fétidos & $\begin{array}{l}\text { Flatos fétidos } \\
\text { Irritabilidad }\end{array}$ \\
\hline Criterios incluyentes & No presenta & No presenta & No presenta \\
\hline $\begin{array}{l}\text { Criterios exclusivos } \\
\text { IgA EmA } \\
\text { IgA Ttg } \\
\text { IgG DPG } \\
\text { IgG AGA }\end{array}$ & $\begin{array}{l}\text { Negativo } \\
\text { Negativo } \\
\text { Negativo } \\
\text { Negativo }\end{array}$ & $\begin{array}{l}\text { Negativo } \\
\text { Negativo } \\
\text { Negativo } \\
\text { Positivo }\end{array}$ & $\begin{array}{l}\text { Negativo } \\
\text { Negativo } \\
\text { Negativo } \\
\text { Negativo }\end{array}$ \\
\hline Genético HLA DQ2-DQ8 & Negativo & Negativo & Pendiente \\
\hline Histología & Normal & Normal & Normal \\
\hline CELIARED & 12 puntos & 27 puntos & 22 puntos \\
\hline Rast & Negativo & Negativo & Clase 2 para leche \\
\hline $\begin{array}{l}\text { Respuesta al } \\
\text { tratamiento }\end{array}$ & $\begin{array}{l}\text { Inicio: peso Pc } 25-50 \\
\text { Final: peso Pc } 75\end{array}$ & $\begin{array}{c}\text { Inicio: peso }<\text { Pc } 3 \\
\text { Final: peso Pc } 10\end{array}$ & $\begin{array}{l}\text { Inicio: peso Pc }<3 \\
\text { Final: peso Pc } 25\end{array}$ \\
\hline
\end{tabular}

* La serología utilizada es para DPG ELISA INOVA (valor de corte mayor de 20), tTg ELISA INOVA (valor de corte mayor de 20), EmA esófago distal de mono, inmunofluorescencia INOVA (valor de corte dilución mayor de 1/5).

* Genético: técnica PCR-ASO reversa para la detección de alelos HLA 2.5 y DQ8.

* Histología: clasificación de Drut-Cueto Rúa. ${ }^{11,12}$

* Rast: IgE específica para proteína de leche, huevo, trigo, maní y pescado.

* Respuesta a la dieta: se valoró con la disminución de la sintomatología clínica y el progreso de peso.

* CELIARED: se trata de un puntaje que se utiliza para valorar la probabilidad diagnóstica de EC. Se lleva a cabo en el Ministerio de Salud de la Provincia de Buenos Airess a través del Programa Provincial de Celiaquía. ${ }^{4}$

EMA: Antiendomisio.

tTG: antitransglutaminasa.

DPG: péptido deaminado de gliadina.

AGA: antigliadina de primera generación. 
Las manifestaciones clínicas de la sensibilidad al gluten incluyen un amplio abanico de síntomas y signos clínicos, además de la variedad de síntomas digestivos con los que se puede presentar (vómitos, diarrea, estreñimiento, distensión abdominal, meteorismo, dispepsia, etc.). La gravedad o intensidad de los síntomas es variable.

Los mecanismos moleculares no son bien conocidos, aunque publicaciones recientes sugieren el papel de la inmunidad innata como una de sus principales causas, a diferencia de la EC, en la que intervienen tanto la inmunidad innata como la adquirida.

La gliadina se digiere solo parcialmente en el tracto gastrointestinal y produce péptidos resistentes a la digestión. Estos péptidos son capaces de atravesar la barrera epitelial intestinal si la permeabilidad intestinal está aumentada. De este modo, se puede desarrollar una respuesta inmune frente a antígenos ambientales que tienen reacción cruzada con antígenos del huésped; este es el inicio de la enfermedad celíaca. Sin embargo, Sapone et al. ${ }^{6}$ han demostrado, en pacientes con SG, una permeabilidad intestinal normal, al encontrar niveles más altos de claudina-4 en las biopsias de pacientes con SG que en las de celíacos y una relación urinaria lactulosa/ manitol elevada en pacientes celíacos y no en aquellos con SG en el test de lactulosa-manitol. Sapone et al. ${ }^{6}$ han descrito, en pacientes con SG comparados con pacientes celíacos, una expresión normal de claudina-1 y ZO-1 y niveles más altos del receptor toll-like 2 y del número de linfocitos intraepiteliales de las clases $\alpha$ y $\beta$, sin aumento en la expresión de genes relacionados con la inmunidad adaptativa en la mucosa intestinal, como IL-6, IL-21 e INF- $\gamma$.

Asimismo, Fabri et al. ${ }^{7,8}$ han demostrado niveles mayores del estimulador de linfocitos B, un marcador sobreexpresado en enfermedades autoinmunes y linfoproliferativas, en pacientes celíacos comparados con pacientes con SG, en los que eran similares a controles sanos. Estos cambios podrían sugerir un importante papel de la inmunidad innata en la $S G$, sin que esté incluida la inmunidad adaptativa. ${ }^{9}$

Por el momento, no hay pruebas específicas de diagnóstico para la sensibilidad al gluten no celíaca, por lo que este requiere descartar primero la celiaquía (o cualquier otra patología que se sospeche, incluida la alergia al trigo) y hacer, después, una dieta sin gluten para verificar si el paciente se recupera. En caso afirmativo, se recomienda reintroducir temporalmente el gluten en su alimentación para comprobar si hay recaída. Si es así, será diagnosticado como sensible al gluten no celíaco. Se aconseja probar la dieta sin TACC por, al menos, cuatro semanas. ${ }^{10}$

En esta serie de pacientes, todos presentaron los síntomas clásicos del cuadro malabsortivo. En nuestro servicio, realizamos, en forma protocolizada, un tratamiento antiparasitario con el objeto de excluir los parásitos como causa de diarrea crónica frecuente en nuestro medio. Posteriormente, de continuar el cuadro clínico, se solicitan estudios serológicos para descartar EC y alergia al trigo y se realiza la planilla de criterios (CELIARED). CELIARED es una herramienta diseñada en el Programa de Celiaquía del Ministerio de Salud de la Provincia de Buenos Aires, cuya intención es lograr un diagnóstico rápido de todos aquellos individuos sintomáticos que la "padecen" y luego ir en la búsqueda de aquellos en "aparente" estado de salud. Para lograr este objetivo, recordamos las formas clínicas y publicamos una ficha con los criterios mayores o signos (cada uno con un valor de 4 puntos), los criterios menores o síntomas (cada uno con un valor de 3 puntos), criterios incluyentes, tales como las enfermedades asociadas o factores de riesgo, como parentesco en primer o segundo grado (cada uno con un valor de 4 puntos), y criterios exclusivos, tales como los marcadores serológicos de alto grado de sensibilidad y especificidad (cada uno con un valor de 35 puntos). Cuando el puntaje supera los 24 puntos, la probabilidad de padecer EC es del $50 \%{ }^{4}$ (Figura 1).

En nuestro Servicio, se utiliza la clasificación Drut-Cueto Rúa para valorar la atrofia intestinal. En esta serie de pacientes, resultaron biopsias de intestino normal. ${ }^{11-12}$

Al no obtener respuesta y luego de excluir las causas más frecuentes de síndrome malabsortivo, se realizó una prueba de exclusión-provocación con una dieta sin TACC. Esta demostró una respuesta positiva.

Los genotipos HLA-DQ2 y/o HLA-DQ8 son positivos en el $40 \%$ de los pacientes. Esta prevalencia es comparable a la de la población general (aproximadamente, el 30\%) y mucho menor que la de la EC (99\%). Por tanto, no parece que la SG esté relacionada genéticamente, aunque no se puede descartar que los pacientes con HLADQ2 o HLA-DQ8 que no tienen en la actualidad sintomatología relacionada con el gluten no desarrollen EC o SG en el futuro. 


\section{CONCLUSIONES}

La sensibilidad al gluten es una entidad para tener en cuenta en aquellos pacientes con síntomas digestivos en los que no se encuentra su causa en el marco de una enfermedad celíaca. Se entiende que la dieta sin TACC representa una mejoría clínica en estos pacientes.

\section{REFERENCIAS}

1. Leonard MM, Vasagar B. US perspective on gluten-related diseases. Clin Exp Gastroenterol 2014;7:25-37.

2. Cooper BT, Holmes GK, Ferguson R, Thompson RA, et al. Gluten-sensitive diarrhea without evidence of celiac disease. Gastroenterology 1980;79(5 Pt 1):801-6.

3. Cueto Rúa E, Drut R, Peña AS. Non-celiac wheat sensitivity is not a new entity. Am J Gastroenterol 2013;108(11):1805.

4. Cueto Rúa E, Guzmán L. Guías de diagnóstico temprano celiaquía. La Plata: Ministerio de salud de la Provincia de buenos aires; abril de 2010 [Disponible en http://www. ms.gba.gov.ar/wp-content/uploads/2013/03/celiaquiadiagnostico-temprano.pdf].

5. Sapone A, Bai JC, Ciacci C, Dolinsek J, et al. Spectrum of gluten-related disorders: consensus on new nomenclature and classification. BMC Med 2012;10:13.

6. Sapone A, Lammers KM, Casolaro V, Cammarota M, et al. Divergence of gut permeability and mucosal inmune gene expression in two gluten-associated conditions: celiac disease and gluten sensivity. BMC Med 2011,9:23.

7. Fabris M, Visentini D, De re V, Picierno A, et al. Elevated B cell-activating factor of the tumour necrosis factor family in coeliac disease. Scand J Gastroenterol 2007;42(12):1434-9.

8. Fabris M,DeVitaS, VisentiniD,FabroC, etal. B-Lymphocyte stimulator and a proliferation-inducing ligand serum levels in IgA-deficient patients with and without celiac disease. Ann N Y Acad Sci 2009;1173:268-73.

9. Díaz Marugán V, Magallares García L, Fernández Caamaño B, Alcolea Sánchez A, et al. ¿Puede ser el gluten perjudicial en pacientes no celiacos? Evid Pediatr 2013;9:1.

10. Fasano A, Catassi C, Sapone A, Sanders D, et al. El primer documento internacional sobre la definición de la sensibilidad al gluten. Conferencia de consenso sobre la sensibilidad al gluten. Londres, 11 y 12 de febrero de 2011. Disponible en: http://www.drschaer-institute. com/smartedit/documents/download/att147020_es.pdf. [Acceso: 23 de octubre de 2014].

11. Drut R, Cueto Rua E. A 1985: Análisis cuantitativos e inmunohistoquímico de la mucosa yeyunal de niños con enfermedad celiaca y con dieta libre de gluten. Arch Argent Pediatr 1985;83(1):20-4.

12. Comité Nacional de Gastroenterología de la Sociedad Argentina de Pediatría. Jornadas Nacionales de Intestino Delgado. Conclusiones: Histopatología, Clínica e Inmunología. Arch Argent Pediatr 1986;84(1):38-9. 